\title{
Treatment of Pain Due to Winged Scapula with Spinal Cord Stimulation: A Case Report
}

\author{
Akshay Bhatt, MD¹, Daniel Taylor, MD², and Russell Bell, MD
}

Background: Winged scapula (WS) is a protrusion of the scapula giving the appearance of a wing and can be due to either a musculoskeletal or neurological insult. Pain associated with WS is multifactorial, including myofascial and neuronal mechanisms, and may be difficult to treat.

Case Report: In a case of WS refractory to conservative management with physical therapy and medications and not a candidate for nerve repair, we treated the pain with a spinal cord stimulator. We report significant pain reduction and improvement in function with the procedure.

Conclusion: This case suggests a role for neuromodulation in WS and requires further investigation.

Key words: Winged scapula, spinal accessory nerve injury, spinal cord stimulation, neuromodulation

\section{BACKGROUND}

Winged scapula (WS) is a protrusion of the medial or lateral border of the scapula giving the appearance of a wing (1). The etiology of WS can be due to either a musculoskeletal or neurological insult resulting in weakness or paralysis of the scapular stabilizers (1). Although WS is primarily a clinical diagnosis, electromyography and nerve conduction studies can help localize the injury (1). Associated pain and weakness can range from none resulting in an aesthetic defect to significant functional impairment of the shoulder (2). Pain may present along the neck, shoulder, and upper back (2) and may include neuronal and myofascial mechanisms $(3,4)$. Severity and etiology of WS guide its treatment and most often alleviates associated pain and weakness. Treatment ranges from conservative management with physical therapy and medications, to surgical interventions to realign the scapula or reconstruct the injured nerve (5). To the best of our knowledge, this is the first report of successful treatment of pain due to WS treated with spinal cord stimulation (SCS).

\section{CASE}

A 38-year-old woman with a history of excised branchial cyst complicated by left spinal accessory nerve injury resulting in WS, a current everyday smoker with generalized anxiety disorder and migraines presented to the pain management clinic with a chief concern of pain and weakness in the left neck and shoulder. No contributory family history was noted.

Symptoms began after a left neck dissection to remove a branchial cyst in 2016 that was complicated by injury to the left spinal accessory nerve. She subsequently developed WS associated with left-sided neck and shoulder pain. Electromyography at the time demonstrated complete denervation of the left trapezius muscle. She was initially managed conservatively with physical therapy and medications, which over the course of 2 years broadened to include: diclofenac $75 \mathrm{mg}$ oral daily, tizanidine $6 \mathrm{mg}$ oral up to 3 times daily as needed for muscle spasm, pregabalin $200 \mathrm{mg}$ oral twice daily, topiramate $25 \mathrm{mg}$ oral twice daily, duloxetine $30 \mathrm{mg}$

From: 'Department of Anesthesia and Critical Care, Division of Pain Medicine, University of Pennsylvania Health System, Philadelphia, PA; ${ }^{2}$ Department of Anesthesia and Critical Care, University of Pennsylvania Health System, Philadelphia, PA

Corresponding Author: Akshay Bhatt, MD, E-mail: akshay.bhatt@pennmedicine.upenn.edu

Disclaimer: There was no external funding in the preparation of this manuscript.

Conflict of interest: Each author certifies that he or she, or a member of his or her immediate family, has no commercial association (i.e., consultancies, stock ownership, equity interest, patent/licensing arrangements, etc.) that might pose a conflict of interest in connection with the submitted manuscript.

Accepted: 2021-04-19, Published: 2021-06-14 
oral daily, and tramadol $50 \mathrm{mg}$ oral every 6 hours as needed for pain. By 2018, there was limited recovery of the left trapezius muscle's function and symptom relief. Repeat electromyography was unchanged. Neurosurgical evaluation determined that she was not a candidate for nerve reconstruction due to etiology of and length of time since the initial injury and she was referred to orthopedic surgery for evaluation of surgical stabilization of the scapula; however, she declined any further surgical intervention. She instead opted for a referral to pain management for symptom control.

At her initial visit with pain management in early 2018, she described her pain as "aching, shooting, and stabbing" over the left neck and shoulder that worsened with activity. Physical exam was remarkable for decreased range of motion of the left upper extremity with inability to abduct above $90^{\circ}$ associated with winging of the scapula. Tenderness to palpation over the posterior left shoulder and scapula was also noted and thought to have a myofascial contribution. Unfortunately, trigger point injections with local anesthetic provided only minimal relief lasting one day. Similarly, a transcutaneous electrical nerve stimulator provided short-term minimal relief. To treat pain from muscles overcompensating for the lack of trapezius contribution to scapular stabilization, she was scheduled for an ultrasound-guided long thoracic nerve block that provided $50 \%$ pain relief for 2.5 weeks. Repeat long thoracic nerve block 2 months later yielded similar results. Due to postsurgical scarring, a spinal accessory nerve block was not attempted. By late 2018 , she was certified for medical marijuana, which improved analgesia and sleep and alleviated some of her anxiety. Furthermore, it allowed over the course of 2019 the tapering and discontinuation of tramadol. Duloxetine was also rotated to nortriptyline $10 \mathrm{mg}$ oral daily and subsequently venlafaxine $37.5 \mathrm{mg}$ oral daily without any further improvement in pain control.

Ultimately, in the summer of 2020, due to unremitting pain that interfered with daily function, a decision was made to trial an SCS to treat the underlying neuropathic pain. To treat symptoms in a high dermatomal distribution and optimize coverage of the spinal accessory nerve, which has contributions from spinal segments C1-C6, while allowing for a margin of safety, a single SCS lead was threaded to the inferior endplate of the $\mathrm{C2}$ vertebral body. Additionally, the lead was placed midline to decrease the risk of ventral migration. The trial lasted for 7 days, during which time she noted $50 \%$ pain relief until the final day. An x-ray at that time revealed lead migration to the C5 vertebral body and the lead was subsequently removed. Due to a successful trial, she was scheduled for a permanent SCS with a single lead again threaded midline to the inferior endplate of the C2 vertebral body. Neuromodulation was high frequency, nonparesthesia-based. On 3-month and 6-month follow-up she reported satisfaction with the permanent SCS placement. She noted greater than $50 \%$ pain relief and improvement in the ability to perform activities of daily living despite continued limitation of left arm abduction. There were no further changes in medications after treatment with SCS.

\section{DISCUSSION}

As illustrated by our case, pain associated with WS may be multifactorial and difficult to treat. WS of neurologic etiology occurs due to injury anywhere along the course of the nerves innervating the scapular stabilizing muscles, including the long thoracic nerve, spinal accessory nerve, and dorsal scapular nerve (1). More proximally, cervical nerve root injury may also cause WS and relief of symptoms after foraminotomy has been described (6). While the primary nerve injury can result in sensitization and stimulation of nociceptors within the myelin sheath, spasm from overcompensating periscapular muscles and impingement of surrounding sensory nerves may also contribute to pain (3). Furthermore, the misaligned scapula may cause tension on the shoulder girdle or direct irritation and compression of the surrounding structures during movement leading to pain (4).

Initial management of mild symptoms involves physical therapy that aims to strengthen compensating periscapular muscles and limit winging of the scapula (2). Similarly, use of a shoulder orthosis to support the scapula in 20 of 28 patients with trapezius palsy after neck dissection was reported to result in pain relief 3 months postsurgery, though with limited arm abduction (7). For patients failing conservative management, surgical interventions to stabilize the scapula may be considered. The Eden-Lange procedure and pectoralis major transfer involve muscle reattachment to improve scapular alignment and have shown favorable results (5). Surgical stabilization of the scapula may also be accomplished by fixation to the rib cage, though a high complication rate has been reported including some that are pulmonary-related, hardware failure, failure of bone fusion, and persistent pain (5). Finally, WS may be 
treated with repair of the damaged nerve by neurolysis, end-to-end suturing, or nerve grafting (8). In a study of spinal accessory nerve repair, severity of damage, as evidenced by intraoperative nerve action potential recordings, guided type of repair and correlated with postoperative outcomes (8). The highest frequency of recovery was reported in patients with positive nerve action potentials undergoing neurolysis (8).

However, as our case demonstrates, for patients who have failed conservative management and are not surgical candidates, SCS may be considered to treat pain associated with WS. SCS generates an electrical field that can range in frequency and duration between metal contacts placed in the epidural space proposed to activate nonnociceptive $A \beta$ fibers that may supersede and inhibit transmission from nociceptive $A \delta$ and $C$ fibers, termed the gate control theory, though other spinal segmental and cortical mechanisms may also play a role (9). Strong evidence supports use of SCS for axial back pain with or without radicular pain and complex regional pain syndrome (10). Studies of SCS in peripheral neuropathic pain syndromes have reported mixed results. In a case of postthoracotomy syndrome recalcitrant to medication management, treatment with SCS resulted in resolution of pain and allodynia (11). A review of treatment of postherpetic neuralgia with SCS reported $77.8 \%$ long-term pain relief in temporary SCS versus $47.1 \%$ in permanent SCS this is thought to be due to delayed placement of the permanent SCS leading to development of central sensitization (12). Analogously, failure of SCS in treatment for phantom limb pain is thought to be due to utilization of neuromodulation as a last resort in severe cases (13).

\section{CONCLUSION}

In adding to the census of treatment for pain due to peripheral nerve injury, we report significant pain relief and improved function in a patient with WS treated with SCS. The lack of complete symptom resolution may be due to the complexity of pain mechanisms in WS including those not well treated by SCS. Further research is required to elucidate the role of neuromodulation in WS. The failure of SCS may also be due to device complication or inappropriate patient selection (14). While growing literature continues to support the use of SCS, further investigation is required to determine appropriate indication and patient selection to optimize treatment success of neuropathic pain conditions with SCS.

\section{Author Contributions}

$A B$ : This author prepared the manuscript.

TH: This author contributed to the manuscript and gathered necessary information for the manuscript.

RB: This author contributed to the manuscript and provided guidance.

\section{REFERENCES}

1. Didesch JT, Tang P. Anatomy, Etiology, and management of scapular winging. J Hand Surg Am 2019; 44:321-330.

2. Meininger AK, Figuerres BF, Goldberg BA. Scapular winging: An update. J Am Acad Orthop Surg 2011; 19:453-462.

3. Sultan HE, Younis El-Tantawi GA. Role of dorsal scapular nerve entrapment in unilateral interscapular pain. Arch Phys Med Rehabil 2013; 94:1118-1125

4. Kibler WB, Sciascia A, Wilkes T. Scapular dyskinesis and its relation to shoulder injury. J Am Acad Orthop Surg 2012; 20:364-372.

5. Vetter M, Charran O, Yilmaz E, et al. Winged scapula: A comprehensive review of surgical treatment. Cureus 2017; 9:e1923.

6. Ishihama $Y$, Tezuka F, Manabe $\mathrm{H}$, et al. Minimally invasive cervical micro-endoscopic foraminotomy for C6 radiculopathy with overlooked scapular winging - A case report. J Med Invest 2019; 66:340-343.

7. Kizilay A, Kalcioglu MT, Saydam L, Ersoy Y. A new shoulder orthosis for paralysis of the trapezius muscle after radical neck dissection: A preliminary report. Eur Arch Otorhinolaryngol 2006; 263:477-480.

8. Kim DH, Cho YJ, Tiel RL, Kline DG. Surgical outcomes of 111 spi-

nal accessory nerve injuries. Neurosurgery 2003; 53:1106-1112; discussion 1102-1103.

9. Sdrulla AD, Guan Y, Raja SN. Spinal cord stimulation: Clinical efficacy and potential mechanisms. Pain Pract 2018; 18:1048-1067.

10. Deer TR, Grider JS, Lamer TJ, et al. A systematic literature review of spine neurostimulation therapies for the treatment of pain. Pain Med 2020; 21:1421-1432.

11. Graybill J, Conermann T, Kabazie AJ, Chandy S. Spinal cord stimulation for treatment of pain in a patient with post thoracotomy pain syndrome. Pain Physician 2011; 14:441-445.

12. Kurklinsky S, Palmer SC, Arroliga MJ, Ghazi SM. Neuromodulation in postherpetic neuralgia: Case reports and review of the literature. Pain Med 2018; 19:1237-1244.

13. Corbett $M$, South $E$, Harden $M$, et al. Brain and spinal stimulation therapies for phantom limb pain: A systematic review. Health Technol Assess 2018; 22:1-94.

14. Atkinson L, Sundaraj SR, Brooker $C$, et al. Recommendations for patient selection in spinal cord stimulation. J Clin Neurosci 2011; 18:1295-1302. 
